\title{
COMPLEX INTERPOLATION AND CONVEXITY
}

\author{
MARCO VIGNATI
}

\begin{abstract}
The relations between the complex theory of interpolation for families of Banach spaces and the notion of uniform convexity are studied. It is proven that the moduli of uniform convexity vary smoothly with the interpolation spaces. A new notion of "distance" between Banach spaces is introduced.
\end{abstract}

1. Introduction and notations. In this paper we investigate the relations between the complex method of interpolation for families of Banach spaces, introduced in [CCRSW], and the notion of uniform convexity.

In $\S 2$ we obtain a generalization of a result due to $\mathrm{M}$. Cwikel and $\mathrm{S}$. Reisner (see $[\mathbf{C w R e}]$ ), stating that the interpolation spaces $A_{s}=\left[A_{0}, A_{1}\right]_{s}, 0<s<1$, obtained using the complex method of A. P. Calderón [Cal], are uniformly convex, provided that at least one of the spaces $A_{0}, A_{1}$ is uniformly convex. In the case of a family $\left\{A_{e^{i \theta}}\right\}, 0 \leq \theta<2 \pi$, of Banach spaces, we obtain estimates for the moduli of uniform convexity of the interpolation spaces, provided that the boundary spaces are uniformly convex when $\theta$ ranges in a subset $U$ of $[0,2 \pi)$ with positive measure. Our result includes the one in [CwRe] and the estimates that we obtain are essentially the best possible.

In $\S 3$ we give estimates that say that the moduli of uniform convexity of complex interpolation families must vary smoothly with the spaces. As a corollary to this result we answer a question posed by $R$. Rochberg in $[\mathbf{R o}]$.

In $\S 4$ we introduce a new concept of "distance" between Banach spaces, involving the complex method of interpolation. The estimates in Theorem 3.1 can be used to show how this "distance" relates to the moduli of uniform convexity of the spaces involved.

Throughout this paper $D$ will denote the open unit disk in the complex plane, $\partial D$ its boundary and $T$ the interval $[0,2 \pi)$. For $z$ in $D$, the Poisson kernel at $z$ is $P_{z}(\theta)=\left(1-|z|^{2}\right) / 2 \pi\left|z-e^{i \theta}\right|^{2}$. By $U$ we always denote some measurable subset of $T$, and $|U|_{z}=\int_{U} P_{z}(\theta) d \theta$ is its harmonic measure.

A complex interpolation family (c.i.f.) of Banach spaces (B-spaces) $\left\{A_{e^{i \theta}}\right\}$ defined on $\partial D$ will also be denoted by $\left\{A_{\theta},|\cdot|_{\theta}\right\}$ and $A[z]$ will be the interpolation space at $z \in D$ constructed in [CCRSW].

If $f, g$ are nonnegative nondecreasing functions defined on some interval $[0, \alpha)$, we say that $f<<g(g$ dominates $f$ at 0$)$ if there exist $a, b>0$ such that $f(t) \leq a g(b t)$ for every $t$ small enough. If $f<<g$ and $g<<f$ we write $f \stackrel{0}{\simeq} g$ ( $f$ and $g$ are equivalent at 0$)$.

Received by the editors October 16, 1985 and, in revised form, February 7, 1986.

1980 Mathematics Subject Classification (1985 Revision). Primary 46B20. 
2. Uniform convexity. Let $(E,|\quad|)$ be a B-space; its modulus of uniform convexity is defined, for $0 \leq \varepsilon \leq 2$, by

$$
\delta_{E}(\varepsilon) \equiv \operatorname{Inf}\{1-\|(x+y) / 2\|:\|x\|,\|y\| \leq 1,\|x-y\| \geq \varepsilon\} .
$$

If $\delta_{E}(\varepsilon)>0$ for all $\varepsilon>0, E$ is said to be a uniformly convex B-space. In this case $\delta_{E}$ is equivalent at 0 to a strictly increasing Orlicz function $\bar{\delta}_{E}$ (see $[\mathbf{L i T z}]$ ). The spaces $L^{p}$ and $l^{p}$ are uniformly convex for $1<p<\infty$, with $\delta_{p}(\varepsilon) \stackrel{0}{\simeq} \varepsilon^{p}$ if $p \geq 2$ and $\delta_{p}(\varepsilon) \stackrel{0}{\simeq} \varepsilon^{2}$ if $1<p \leq 2$.

In [CwRe] the authors prove that if $\left(A_{0}, A_{1}\right)$ is a compatible pair of B-spaces and $A_{s} \stackrel{\prime}{=}\left[A_{0}, A_{1}\right]_{s}, 0<s<1$, are the spaces obtained by the complex method of interpolation of $\mathrm{A}$. P. Calderón (see $[\mathbf{C a l}]$ ), then the spaces $A_{s}$ are uniformly convex, provided that at least one of $A_{0}, A_{1}$ has the same property. Similar results concerning the real interpolation spaces have been obtained by Beauzamy (see $[\mathbf{B}]$ ). In [CwRe] it is shown that if $A_{0}$ (or $\left.A_{1}\right)$ is uniformly convex, then $\delta_{0}\left(\varepsilon^{1 /(1-s)}\right)<<$ $\delta_{s}(\varepsilon)$ (or $\delta_{1}\left(\varepsilon^{1 / s}\right)<<\delta_{s}(\varepsilon)$ ); and if both $A_{0}, A_{1}$ are uniformly convex, then

$$
\left[\left(\delta_{0}^{-1}\right)^{1-s}\left(\delta_{1}^{-1}\right)^{s}\right]^{-1}<<\delta_{s} .
$$

The corresponding version in the case of a c.i.f. $\left\{A_{\theta}\right\}$ is

THEOREM 2.2. Let $\left\{A_{\theta}\right\}$ be a c.i.f. of B-spaces, defined on $\partial D$; suppose $z \in D$ and $U \subset T$ with $|U|_{z}>0$. If the spaces $A_{\theta}$ are uniformly convex for $\theta \in U$, then

$$
\psi_{z} \equiv\left[\exp \int_{U} \log \delta_{\theta}^{-1} p_{z}(\theta) d \theta\right]^{-1}<<\delta_{z}
$$

where $\delta_{\theta}=\delta_{A_{\theta}}$ and $\delta_{z}=\delta_{A[z]}$.

REMARKS. (i) It is clear that if $A_{\theta}=A_{0}$ for $\theta \in U$ and $A_{\theta}=A_{1}$ for $\theta \notin U$ we recapture the result of $[\mathbf{C w R e}]$.

(ii) The case $A_{\theta}=L^{p(\theta)}, 2 \leq p(\theta) \leq \infty$, shows that (2.3) is the best possible result.

In order to prove Theorem 2.2 we need some facts about the complex interpolation of B-lattices, and some preliminary lemmas.

FACT 2.4. Let $(M, d \mu)$ be a measure space and $\left\{X_{\theta},\|\cdot\|_{\theta}\right\}$ a family of Blattices of functions over $M$, defined for each $\theta \in T$. If $\left\{X_{\theta}\right\}$ is a c.i.f. of B-spaces and $z \in D$, we can define the class $\left[X_{\theta}\right]^{z}$ of functions $f$ over $M$, for which there exist $\lambda>0$ and $F: T \times M \rightarrow \mathbf{R}$ such that

$$
\begin{gathered}
\|F(\theta, \cdot)\|_{\theta} \leq 1 \text { and } \\
|f(x)| \leq \lambda \exp \int_{0}^{2 \eta} \log |F(\theta, x)| P_{z}(\theta) d \theta \quad \text { a.e. in } M .
\end{gathered}
$$

If we set $\|f\|^{z}=\operatorname{Inf}\{\lambda>0:(2.5)$ holds $\}$ the space $\left(\left[X_{\theta}\right]^{z},\|\cdot\|^{z}\right)$ becomes a B-lattice (see $[$ Her $]$ ).

Now let $E$ be a B-space and $\varphi$ a convex increasing function on $[0, \infty)$ such that $\varphi(0)=0$. By $\varphi(E)$ we denote the B-lattice of all pairs $(a, b) \in E \times E$ by

By $(E \oplus E)_{\infty}$ we denote the B-lattice of all pairs $(a, b) \in E \times E$ with the norm given by $\|(a, b)\|_{(E \oplus E)_{\infty}}=\operatorname{Max}(\|a\|,\|b\|)$. 
For $U \subset T, z \in D$, and $|U|_{z}>0$, let us assume that for each $\theta \in U$ we have a function $\varphi_{\theta}$ as above, such that $\int_{U} \log \varphi_{\theta}^{-1}(t) P_{z}(\theta) d \theta<\infty$ for all $t>0$. Then the function

$$
\varphi_{z}=\left[\exp \int_{U} \log \varphi_{\theta}^{-1} P_{z}(\theta) d \theta\right]^{-1}
$$

is convex increasing, and $\varphi_{z}(0)=0$ (see [Her]).

In this case we have

Proposition 2.8. Let $B_{\theta}=\varphi_{\theta}(E)$ for $\theta \in U$, and $B_{\theta}=(E \oplus E)_{\infty}$ for $\theta \notin U$. Then

$$
\left[B_{\theta}\right]^{z} \subset \bar{\varphi}_{z}(E) \quad \text { (norm decreasing), }
$$

where $\bar{\varphi}_{z}(t)=\varphi_{z}\left(e^{-1 / e}|U|_{z} t\right)$.

Proof. Suppose $\|(x, y)\|^{z} \leq 1$ and fix $\varepsilon>0$. According to (2.5) we can find $c, d: T \rightarrow \mathbf{R}^{+}$such that: $\|x\| \leq(1+\varepsilon)\left(\exp \int_{T} \log c(\theta) P_{z}(\theta) d \theta\right),\|y\| \leq$ $(1+\varepsilon)\left(\exp \int_{T} \log d(\theta) P_{z}(\theta) d \theta\right), \operatorname{Max}[c(\theta), d(\theta)] \leq 1$ if $\theta \notin U$ and $c(\theta)+\varphi_{\theta}^{-1}(d(\theta)) \leq$ 1 if $\theta \in U$. Set $\alpha(\theta)=\varphi_{\theta}(d(\theta)) \chi_{U}(\theta)$ and let

$$
c=\int_{T} c(\theta) P_{z}(\theta) d \theta, \quad \alpha=\int_{T} \alpha(\theta) P_{z}(\theta) d \theta .
$$

Obviously we have $\alpha+c \leq 1$ and

$$
\begin{gathered}
\|x\| \leq(1+\varepsilon) c, \\
\|y\| \leq(1+\varepsilon)\left(\exp \int_{U} \log \varphi_{\theta}^{-1}(\alpha(\theta)) P_{z}(\theta) d \theta\right) .
\end{gathered}
$$

Let $A=\left(\left\{\theta \in U: \alpha(\theta) \leq \alpha|U|_{z}\right\}\right.$ and $B=U-A$. If $|B|_{z}=0$, then $a(\theta)=\alpha|U|_{z}$ for a.e. $\theta \in U$, and so $\|y\| \leq(1+\varepsilon) \varphi_{z}^{-1}\left(\alpha|U|_{z}\right)$. Hence, we have $\alpha+c \leq 1$, $\|x\| \leq(1+\varepsilon) c$, and $\|y\| \leq(1+\varepsilon) \varphi_{z}^{-1}(\alpha)$, which implies, since $\varepsilon$ is arbitrary, that $\|(x, y)\|_{\bar{\varphi}_{z}(E)} \leq\|(x, y)\|_{\varphi_{z}(E)} \leq 1$.

If $|B|_{z}>0$, from the fact that $\varphi_{\theta}^{-1}$ is concave and increasing we obtain

$$
\begin{aligned}
& \varphi_{\theta}^{-1}(\alpha(\theta)) \leq \varphi_{\theta}^{-1}\left(\alpha|U|_{z}\right) \quad \text { when } \theta \in A, \\
& \varphi_{\theta}^{-1}(\alpha(\theta)) \leq \varphi_{\theta}^{-1}\left(\alpha|U|_{z}\right) \alpha(\theta) / \alpha|U|_{z} \quad \text { when } \theta \in B .
\end{aligned}
$$

Hence

$$
\begin{aligned}
\|y\| \leq & (1+\varepsilon)\left(\exp \int_{U} \log \varphi_{\theta}^{-1}\left(\alpha|U|_{z}\right) P_{z}(\theta) d \theta\right) \\
& \times\left(\exp |B|_{z} \int_{B} \log \frac{\alpha(\theta)}{\alpha|U|_{x}} P_{z}(\theta) \frac{d \theta}{|B|_{z}}\right) \\
\leq & (1+\varepsilon) \varphi_{z}^{-1}\left(\alpha|U|_{z}\right)\left(\alpha|B|_{z}|U|_{z}\right)^{-|B|_{z}}\left(\int_{B} \alpha(\theta) P_{z}(\theta) d \theta\right) \\
\leq & (1+\varepsilon) \varphi_{z}^{-1}\left(\alpha|U|_{z}\right)\left(|U|_{z}|B|_{z}\right)^{-|B|_{z}} \\
\leq & (1+\varepsilon) e^{-1 / e}|U|_{z}^{-1} \varphi_{z}^{-1}\left(\alpha|U|_{z}\right) .
\end{aligned}
$$

Thus we have $\alpha+c \leq 1,\|x\| \leq(1+\varepsilon) c, \bar{\varphi}_{z}(\|y\|) \leq(1+\varepsilon) \alpha$, and, as before, $\|(x, y)\|_{\bar{\varphi}_{z}(E)} \leq 1$. 
FACT 2.10. If $\left\{A_{\theta}\right\}$ is a c.i.f. of B-spaces, and $\left\{X_{\theta}\right\}$ is a c.i.f. of B-lattices, then $\left\{X_{\theta}\left(A_{\theta}\right)\right\}$ is a c.i.f. of B-lattices, and

$$
\left[X_{\theta}\left(A_{\theta}\right)\right][z] \rightarrow\left[X_{\theta}\right]^{z}(A[z])
$$

is a norm-decreasing embedding (see $[\mathbf{H e r}]$ ).

LEMMA 2.11. Let $E$ be a B-space, and $\delta$ a nonnegative nondecreasing function defined on some $[0, \alpha)$. Suppose that there exist $a, b>0$ such that

$$
\operatorname{Max}(\|x+y\|,\|x-y\|) \leq 1 \text { implies }\|x\|+a \delta(b\|y\|) \leq 1
$$

for all $x, y \in E$. Then $\delta<<\delta_{E}$.

PROOF. Straightforward, apply definition (2.1).

REMARKS. (i) If $\delta=\delta_{E}(2.12)$ holds with $a=1, b=2$.

(ii) Lemma 2.11 is a weaker version of Lemma 3 in [CwRe]. Here we can avoid the use of the converse implication and so we do not need a result of Pisier.

ProOF OF THEOREM 2.2. Let $S: A_{\theta} \times A_{\theta} \rightarrow A_{\theta} \times A_{\theta}$ be defined by $S(a ; b)=$ $\frac{1}{2}(a+b ; a-b)$. From Lemma 2.11 we can see that, if $\theta \in U, S:\left(A_{\theta} \oplus A_{\theta}\right)_{\infty} \rightarrow \varphi_{\theta}\left(A_{\theta}\right)$ with norm $\eta\left(e^{i \theta}\right) \leq 1$, where $\varphi_{\theta}(t)=\delta_{\theta}(2 t)$.

When $\theta \notin U$ we use the fact, true in every B-space $(E,\|\cdot\|)$, that $S:(E \oplus E)_{\infty} \rightarrow$ $(E \oplus E)_{\infty}$ with norm 1 .

Thus, if $\theta \notin U, S:\left(A_{\theta} \oplus A_{\theta}\right)_{\infty} \rightarrow\left(A_{\theta} \oplus A_{\theta}\right)_{\infty}$ with $\eta\left(e^{i \theta}\right)=1$.

Using $X_{\theta}\left(A_{\theta}\right)=\varphi_{\theta}\left(A_{\theta}\right)$ for $\theta \in U$, and $X_{\theta}\left(A_{\theta}\right)=\left(A_{\theta} \oplus A_{\theta}\right)_{\infty}$ for $\theta \notin U$, we can summarize our result in

$$
S:\left(A_{\theta} \oplus A_{\theta}\right)_{\infty} \rightarrow X_{\theta}\left(A_{\theta}\right) \text { with } \eta\left(e^{i \theta}\right) \leq 1 \text { for } \theta \in T
$$

Now we apply the usual interpolation results and obtain

$$
S:\left\{\left(A_{\theta} \oplus A_{\theta}\right)_{\infty}\right\}[z]=(A[z] \oplus A[z])_{\infty} \rightarrow\left[X_{\theta}\left(A_{\theta}\right)\right][z]
$$

with norm not exceeding 1 .

The use of Fact 2.10 and Proposition 2.8 tells us that $S$ maps $([z] \oplus A[z])_{\infty}$ into $\psi_{z}(A[z])$ with norm not larger than 1 . We now apply Lemma 2.11 and have the proof.

REMARK. We are grateful to S. Dilworth for informing us that a result similar to Theorem 2.2 does not hold for the case of PL-convexity (for the definition and properties see [DGT-J]). There is an example, due to G. Pisier and contained in [Dil], of an interpolation pair $\left(A_{0}, A_{1}\right)$ of B-spaces which are PL-convex, but such that $A_{s}$ is not PL-convex for $0<s<1$.

3. A continuity result for the moduli of uniform convexity. In this section $\left\{A_{\theta}\right\}$ will denote a c.i.f. of B-spaces, defined on $\partial D$, with the property that also the family $\left\{A_{\theta}^{*}\right\}$ of the dual spaces is a c.i.f., and that the duality result holds, i.e. $(A[z])^{*}=\left\{A_{\theta}^{*}\right\}[z]$. Our purpose is to show that the moduli of uniform convexity vary smoothly with $z \in D$. More precisely:

THEOREM 3.1. Let $\left|z_{0}\right|=\rho<1$ and $0 \leq \eta \leq 1-4 \rho$; then

$$
\delta_{z_{0}}(\eta) \leq \delta_{0}(\eta+4 \rho)+2 \rho .
$$

We delay the proof of this theorem and see an application of it. In [Ro], R. Rochberg posed the problem "if $A[0]=l^{3}$ and $A\left[z_{0}\right]=l^{2}$, can $\left|z_{0}\right|$ be arbitrarily small?" The answer comes from the following result. 
COROLlaRY 3.3. If $A[0]=l^{3}$ and $A\left[z_{0}\right]=l^{2}$, then $\left|z_{0}\right|$ cannot be arbitrarily small.

ProOF. From $[\mathbf{L i T z}]$ we get

$$
\delta_{0}(\eta)=1-\left[1-(\eta / 2)^{3}\right]^{1 / 3} \text { and } \delta_{z_{0}}(\eta)=1-\left[1-(\eta / 2)^{2}\right]^{1 / 2} .
$$

So (3.2) becomes

$$
\left[1-(\eta+4 \rho)^{3} / 8\right]^{1 / 3} \leq 2 \rho+\left[1-(\eta / 2)^{2}\right]^{1 / 2},
$$

which implies

$$
h(\eta ; \rho)=(\eta+4 \rho)^{3}-\eta^{2}+16 \rho \geq 0
$$

for every $0 \leq \eta \leq 1-4 \rho, 0 \leq \rho \leq 1$, and it is easy to check that (3.4) cannot be satisfied for all $\eta$ small unless $\rho \geq \rho_{0}$.

PROOF OF THEOREM 3.1. Let $x, y \in A[0]$ such that $|x|_{0}=|y|_{0}=1$ and $|x-y|_{0}=\varepsilon>0$. For $\tau>0$ fixed we can find a "quasi-extremal" function $x(z)$ for $x$ at $z=0$, i.e., an analytic function $x(z)$ such that $x(0)=x$ and $\left|x\left(e^{i \theta}\right)\right|_{\theta} \leq 1+\tau$ for a.e. $\theta \in T$. Similarly, we can find a quasi-extremal function $y(z)$ for $y$ at $z=0$. Moreover, we can find a vector $(x-y)^{*} \in A[0]^{*}$ such that $\left|(x-y)^{*}\right|_{0}^{*}=1$ and $\left\langle x-y,(x-y)^{*}\right\rangle=\varepsilon$. Let $(x-y)^{*}(z)$ be a quasi-extremal function for $(x-y)^{*}$ at $z=0$, and let $\varphi(z)=\left\langle x(z)-y(z),(x-y)^{*}(z)\right\rangle$. The function $\varphi$ is analytic and $\varphi(0)=\varepsilon,\left|\varphi\left(e^{i \theta}\right)\right| \leq 2(1+\tau)^{2}$. Applying the Schwarz-Pick lemma we get

$$
\begin{aligned}
|\varphi(z)| & \geq 2(1+\tau)^{2}\left[\varepsilon-2|z|(1+\tau)^{2}\right]\left[\varepsilon|z|+2(1+\tau)^{2}\right]^{-1} \\
& \geq\left[\varepsilon-2|z|(1+\tau)^{2}\right][1-\varepsilon|z| / 2] \\
& \geq \varepsilon-4(1+\tau)^{2}|z|
\end{aligned}
$$

and so

$$
|x(z)-y(z)|_{z} \geq|\varphi(z)| /(1+\tau) \geq(1+\tau)^{-1}\left[\varepsilon-4(1+\tau)^{2}|z|\right] .
$$

If $u=x\left(z_{0}\right), v=y\left(z_{0}\right)$ we have $u, v \in A\left[z_{0}\right]$ such that $|u|_{z_{0}},|v|_{z_{0}} \leq 1$ and

$$
|u-v|_{z_{0}} \geq \varepsilon(1+\tau)^{-2}-4\left|z_{0}\right| \text {. }
$$

Similarly, we can find a vector $((x+y) / 2)^{*} \in A[0]^{*}$ such that

$$
\left|\left(\frac{x+y}{2}\right)^{*}\right|_{0}^{*}=1 \text { and }\left\langle\frac{x+y}{2},\left(\frac{x+y}{2}\right)^{*}\right\rangle=\left|\frac{x+y}{2}\right|_{0} .
$$

If $((x+y) / 2)^{*}(z)$ is a quasi-extremal function for $((x+y) / 2)^{*}$ at $z=0$, and

$$
\psi(z)=\left\langle\frac{x(z)-y(z)}{2},\left(\frac{x+y}{2}\right)^{*}(z)\right\rangle,
$$

the use of the Schwarz-Pick lemma gives us

$$
\left|\frac{u+v}{2}\right|_{z_{0}} \geq\left|\frac{x+y}{2}\right|_{0}-\left|z_{0}\right|\left[1+(1+\tau)^{2}\right] .
$$

We apply now definition (2.1) and let $\tau \rightarrow 0$ to obtain

$$
\delta_{z_{0}}(\eta) \leq 2 \rho+\delta_{0}(\eta+4 \rho),
$$

where $\left|z_{0}\right|=\rho$ and $\eta+4 \eta=\varepsilon$. 
4. Another application to Theorem 3.1. The result obtained in Theorem 3.1 is related to a new definition of "distance" between B-spaces, recently introduced by R. Rochberg. We are grateful to Professor Rochberg for allowing us to introduce this subject.

If $A, B$ are B-spaces, one can define

$$
\bar{d}(A, B)=\operatorname{Inf} d_{H}\left(z_{1}, z_{2}\right)
$$

where

$$
d_{H}(z, w)=\frac{1}{2} \log \left[\left(1+\left|\frac{z-w}{1-\bar{z} w}\right|\right) /\left(1-\left|\frac{z-w}{1-\bar{z} w}\right|\right)\right]
$$

is the hyperbolic distance between two points $z, w \in D$, and the Infimum is taken over all the interpolation families $\left\{A_{\theta}\right\}$ such that $A\left[z_{1}\right]=A, A\left[z_{2}\right]=B$, and for which the duality result holds. We notice that Corollary 3.3 can be restated by saying that $\bar{d}\left(l^{2}, l^{3}\right)>0$. This result was previously known only for the finitedimensional case.

Obviously $\bar{d}(A, A)=0$ and $\bar{d}(A, B)=\bar{d}(B, A)$. However, the triangle inequality may not make sense. One possible way to overcome this difficulty is to define

$$
d(A, B)=\operatorname{Inf} \sum_{j=1}^{N} \bar{d}\left(A_{j+1}, A_{j}\right)
$$

where $A_{1}=A, A_{N+1}=B$; then the triangle inequality is satisfied, and this makes $d(\cdot, \cdot)$ a semidistance. For the finite-dimensional spaces, the results of $[\mathbf{R o}]$ show that $d(\cdot, \cdot)$ is a distance.

Theorem 3.1 can be used to relate the "distance" $d(A, B)$ to the moduli of uniform convexity of the spaces $A$ and $B$.

If $d=d(A, B)$ and $\tau>0$ is fixed, we can find a finite sequence of B-spaces $A=A_{1}, \ldots, A_{N+1}=B$ such that $d \leq \sum_{j=1}^{N} \bar{d}\left(A_{j+1}, A_{j}\right) \leq d+\tau$.

Moreover, for $\sigma>0$ fixed and any $j=1, \ldots, N$ we can find $z_{j} \in D$ such that $A_{j}=A^{(j)}[0]$ and $A_{j+1}=A^{(j)}\left[z_{j+1}\right]$, where $\left\{A^{(j)}[z]\right\}$ are interpolation families as above, and $\bar{d}\left(A_{j}, A_{j+1}\right) \leq d_{H}\left(0, z_{j}\right) \leq \bar{d}\left(A_{j}, A_{j+1}\right)+\sigma$. Choosing $\sigma<d / N$ we then obtain

$$
\sum_{j=1}^{N} d_{H}\left(0, z_{j}\right) \leq\left[\sum_{j=1}^{N} \bar{d}\left(A_{j}, A_{j+1}\right)\right]+N \sigma \leq 2 d+\tau .
$$

Moreover, from Theorem 3.1 we know that $\delta_{A_{j+1}}(\eta) \leq \delta_{A_{j}}\left(\eta+4\left|z_{j}\right|\right)+2\left|z_{j}\right|$. So we obtain

$$
\delta_{B}(\varepsilon) \leq \delta_{A_{N}}\left(\varepsilon+4\left|z_{N}\right|+2\left|z_{N}\right| \leq \cdots \leq \delta_{A}\left(\varepsilon+4 \sum_{j=1}^{N}\left|z_{j}\right|\right)+2\left(\sum_{j=1}^{N}\left|z_{j}\right|\right) .\right.
$$

Since $|z| / 2 \leq d_{H}(0, z) \leq 2|z|$ for $z$ small, we apply (4.3) to obtain

$$
\delta_{B}(\varepsilon) \leq \delta_{A}\left(\varepsilon+8 \sum_{j=1}^{N} d_{H}\left(0, z_{j}\right)\right)+4 \sum_{j=1}^{N} d_{H}\left(0, z_{j}\right) \leq \delta_{A}(\varepsilon+16 d+8 \tau)+8 d+2 \tau
$$


But $\tau$ is arbitrary, and we can get

COROLlARY 4.4. $\delta_{B}(\varepsilon) \leq \delta_{A}(\varepsilon+c d(A, B))+c^{\prime} d(A, B)$, where $c$, $c^{\prime}$ are absolute constants.

Formula (4.4) relates the distance $d(A, B)$ to the moduli of convexity $\delta_{A}, \delta_{B}$. If we specialize it to the case when $d(A, B)=0$ we obtain $\delta_{b} \leq \delta_{A}$ and, by symmetry, $\delta_{A}=\delta_{B}$. This shows that also for $d(\cdot, \cdot)$ we have $d\left(l^{2}, l^{3}\right)>0$.

ACKNOWLEDGMent. I would like to thank Professors R. Rochberg and G. Weiss for introducing me to the subject and the problems studied in the paper, as well as for their useful advice during the course of the research.

\section{REFERENCES}

[B] B. Beauzamy, Espaces d'interpolation réels: topologie et géométrie, Lecure Notes in Math., vol. 666, Springer-Verlag, 1978.

[Ca] A. P. Calderon, Intermediate spaces and interpolation, the complex method, Studia Math. 24 (1964), 113-190.

[CCRSW] R. Coifman, M. Cwikel, R. Rochberg, Y. Sagher, and G. Weiss, A theory of complex interpolation for families of Banach spaces, Adv. in Math. 43 (1982), 203-229.

[CwRe] M. Cwikel and S. Reisner, Interpolation of uniformly convex Banach spaces, Proc. Amer. Math. Soc. 84 (1982), 555-559.

[DGT-J] W. Davis, D. Garling, and N. Tomczak-Jaegermann, The complex convexity of quasinormed linear spaces, J. Funct. Anal. 55 (1984), 110-150.

[Dil] S. J. Dilworth, Complex convexity and the geometry of Banach spaces, Math. Proc. Cambridge Philos. Soc. (to appear).

[Her] E. Hernandez, Intermediate spaces andd the complex method of interpolation for families of Banach spaces, Ann. Mat. Pura Appl. (to appear).

[LiTz] J. Lindenstrauss and L. Tzafriri, Classical Banach spaces. II, Ergeb. Math. Grenzgeb. 97, Springer-Verlag, 1979.

[Ro] R. Rochberg, Function theoretic results for complex interpolation families of Banach spaces, Trans. Amer. Math. Soc. 284 (1984), 745-758. 63130

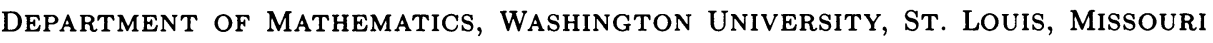

Current address: Dipartimento di Matematica, Politecnico di Torino, 10100 Torino, Italy 